\title{
Using serological measures to monitor changes in malaria transmission in Vanuatu
}

\author{
Jackie Cook*1, Heidi Reid², Jennifer lavro3 ${ }^{3}$, Melissa Kuwahata4 ${ }^{4}$, George Taleo ${ }^{3}$, Archie Clements², James McCarthy4,5, \\ Andrew Vallely² and Chris Drakeley'
}

\begin{abstract}
Background: With renewed interest in malaria elimination, island environments present unique opportunities to achieve this goal. However, as transmission decreases, monitoring and evaluation programmes need increasingly sensitive tools to assess Plasmodium falciparum and Plasmodium vivax exposure. In 2009, to assess the role of serological markers in evaluating malaria transmission, a cross-sectional seroprevalence study was carried out in Tanna and Aneityum, two of the southernmost islands of the Vanuatu archipelago, areas where malaria transmission has been variably reduced over the past few decades.
\end{abstract}

Methods: Malaria transmission was assessed using serological markers for exposure to $P$. falciparum and $P$. vivax. Filter blood spot papers were collected from 1,249 people from Tanna, and 517 people from Aneityum to assess the prevalence of antibodies to two P. falciparum antigens (MSP- $1_{19}$ and AMA-1) and two P. vivax antigens (MSP- $1_{19}$ and AMA-1). Age-specific prevalence was modelled using a simple catalytic conversion model based on maximum likelihood to generate a community seroconversion rate (SCR).

Results: Overall seropositivity in Tanna was 9.4\%, 12.4\% and 16.6\% to $P$. falciparum MSP- ${ }_{19}$, AMA-1 and Schizont Extract respectively and $12.6 \%$ and $15.0 \%$ to $P$. vivax MSP- $1_{19}$ and AMA-1 respectively. Serological results distinguished between areas of differential dominance of either P. vivax or P. falciparum and analysis of age-stratified results showed a step in seroprevalence occurring approximately 30 years ago on both islands, indicative of a change in transmission intensity at this time. Results from Aneityum suggest that several children may have been exposed to malaria since the 2002 P. vivax epidemic.

Conclusion: Seroepidemiology can provide key information on malaria transmission for control programmes, when parasite rates are low. As Vanuatu moves closer to malaria elimination, monitoring changes in transmission intensity and identification of residual malaria foci is paramount in order to concentrate intervention efforts.

\section{Background}

Malaria elimination is once again a stated goal for a number of malaria-endemic countries [1]. In areas where transmission intensity is low, malaria control programmes are considering switching from sustained control to elimination. Monitoring of malaria transmission intensity (MTI), and targeting interventions to settlements experiencing higher malaria transmission will be vital in this endeavour [2]. Interventions in geographically isolated areas, such as islands, may be more success-

\footnotetext{
* Correspondence: Jackie.Cook@lshtm.ac.uk

1 London School of Hygiene \& Tropical Medicine, Keppel Street, London WC1E 7HT, UK

Full list of author information is available at the end of the article
}

ful due to better control of imported transmission, geographically defined areas to target, and high community participation $[3,4]$. Sustained control efforts and monitoring in these areas can lead to the successful eradication of malaria, such as occurred in Mauritius in the 1960s [5].

However, areas of low transmission, or areas where transmission has been reduced substantially, pose considerable challenges for monitoring and evaluation. Exposure to infection can be markedly heterogeneous which can be an important determinant of the rate of progress, and thus the time required to achieve elimination [6]. When transmission is low, traditional measures of MTI such as the entomological inoculation rate (EIR) and par- 
asite rates (PR) lack sensitivity because numbers of positive samples (mosquito and human) are low. Additionally, the low frequency of positive samples represents a formidable logistical challenge, and further, both entomological and parasitological measures are affected by seasonality [7-9]. An alternative measure for MTI is to calculate the prevalence of anti-malaria antibodies in the local population. Serological markers of transmission show greater sensitivity in low transmission areas, as seroprevalence reflects cumulative exposure and thus is less affected by seasonality due to the longer duration of specific antibody responses. Sample collection and analysis can be readily scaled up, and analysis is relatively simple, making this approach adaptable for resource poor settings [10].

Seroepidemiological studies have previously been used to assess malaria transmission intensity [11], reductions in transmission [12-14] and malaria eradication [5,15,16]. However, variation in source of antigen and the subjectivity of the detection methods led to this method falling out of favour [17]. The availability of specific recombinant malarial antigens, and the development of standardized, sensitive enzyme-linked immunosorbent assays (ELISA) mean that seroepidemiological study has once again become an attractive methodology, both for assessment of malaria transmission and for assessing changes in prevalence following the implementation of control programmes [10,18-21].

The utility of serological markers as a methodology for describing malaria exposure was investigated as part of the evaluation of malaria elimination efforts in Vanuatu. Vanuatu is a South Pacific archipelago made up of over 80 islands, each with varying levels of malaria transmission. There have been several large-scale control programmes implemented in the country over the last 50 years and a reduction in reported cases has been recorded over the last decade (Ministry of Health, Vanuatu). In March 2008, the Vanuatu government revised the goal of their National Malaria Programme (NMP) from control to elimination, with the aim to eliminate malaria from Tafea, the most southerly province, by 2015 (Figure 1). The most southerly island of the province, Aneityum (Population $c .800$ ), has been subject to extensive malaria control programmes. There were no reported malaria cases on Aneityum between 1991 and 2002, when a P. vivax epidemic occurred [3]. Since 2002, no more cases have been reported. Tanna, the second largest of the islands in the province, (Population c.20,000) is characterized by low but continuing $P$. falciparum and $P$. vivax transmission. A malariometric study performed in 2008 revealed low level parasitaemia and mainly coastal transmission for both species [22].

This paper reports on the sero-epidemiological results from samples collected from Tanna and Aneityum in May
2009 , with the aim of assessing the utility of specific serological tools for both ongoing monitoring of MTI, and assessment of changes in MTI, in the pre- and post-elimination era.

\section{Methods \\ Study site and population}

Tanna and Aneityum are in Tafea Province, in southern Vanuatu (Figure 1). The main occupation in the province tends to be subsistence farming which takes place year round. Group meetings take place in Nakamals (central meeting place), and men often convene (often in the evenings) to drink kava (a sedating drink) in these areas. Knowledge of malaria is predominantly high, although community adherence to protective measures can be low where the threat is perceived as minimal[23]. Rainfall is seasonal, with a wet season from January to May. The only known malaria vector is Anopheles farauti [3]. In 1988, country-wide distribution of insecticide-treated nets (ITNs) began, following the cessation of indoor residual spraying (IRS) [24].

\section{Tanna}

Tanna has a population of approximately 20,000 (1999 census). Both $P$. falciparum and $P$. vivax malaria occur on Tanna. In 2008, a prevalence study using species specific PCR of samples obtained from school children identified three main areas of malaria transmission [22] (Figure 2). Briefly, Northern Tanna (Hebron/Lounapil) had the highest malaria prevalence with $2.1 \%(10 / 486)$ and $4.7 \%(23 /$ 486) positive for $P$. falciparum and $P$. vivax respectively; the Northern highlands had the lowest with 2.5\% (12/ $481)$ and $1.9 \%(9 / 481)$ for $P$. falciparum and $P$. vivax respectively; and the Southern Coast of Tanna (Etakua/ Kwamera) had very low levels of $P$. falciparum $(0.5 \%, 3 /$ 576) but higher levels of $P$. vivax $(5.0 \%, 29 / 576)$. These figures are summarized in Figure 2. Communities living in these settlements were asked to participate in the seroepidemiological study.

\section{Aneityum}

Aneityum lies to the south of Tanna and is much smaller. The island is sparsely inhabited with a population of approximately 800 distributed in three main settlements. Due to weather constraints during the survey, samples were only collected from two of the three settlements; Anel and Port Patrick. Results for these two settlements have been combined for the majority of the analyses. In Aneityum, weekly mass drug administration (using Primaquine with chloroquine and pyrimethamine with sulphadoxine) was carried out for 9 weeks in 1991. Larvivorous fish were simultaneously introduced into identified breeding sites of An. farauti [3]. No parasites have been detected by microscopy in annual surveys since 2003 (A. Kaneko; personal communication). 


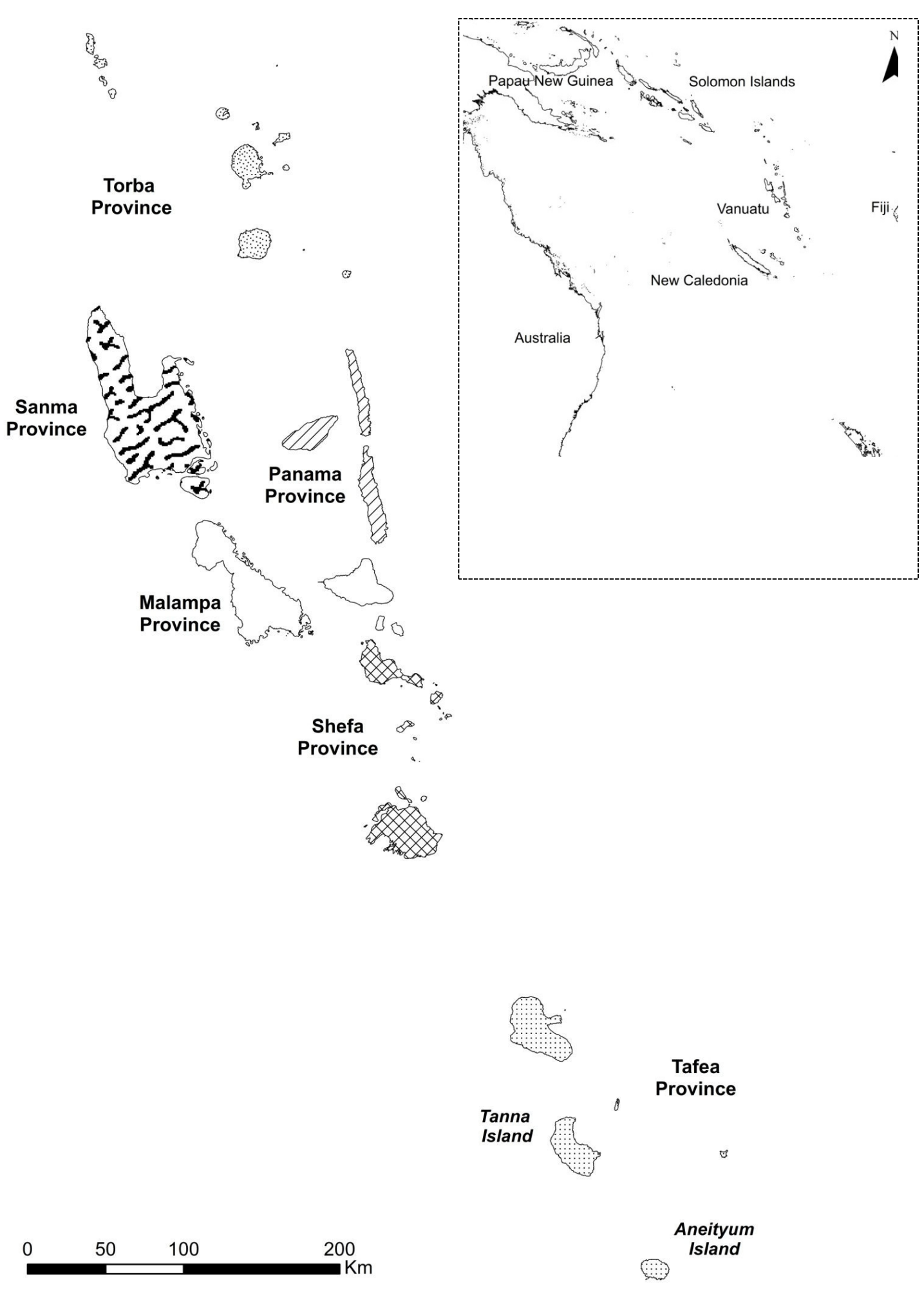

Figure 1 Location of Tanna and Aneityum, Vanuatu in the South West Pacific. Map of Vanuatu showing the location of Tafea Province within the country and the location of Vanuatu with respect to neighbouring countries in the Western Pacific region (inset). 


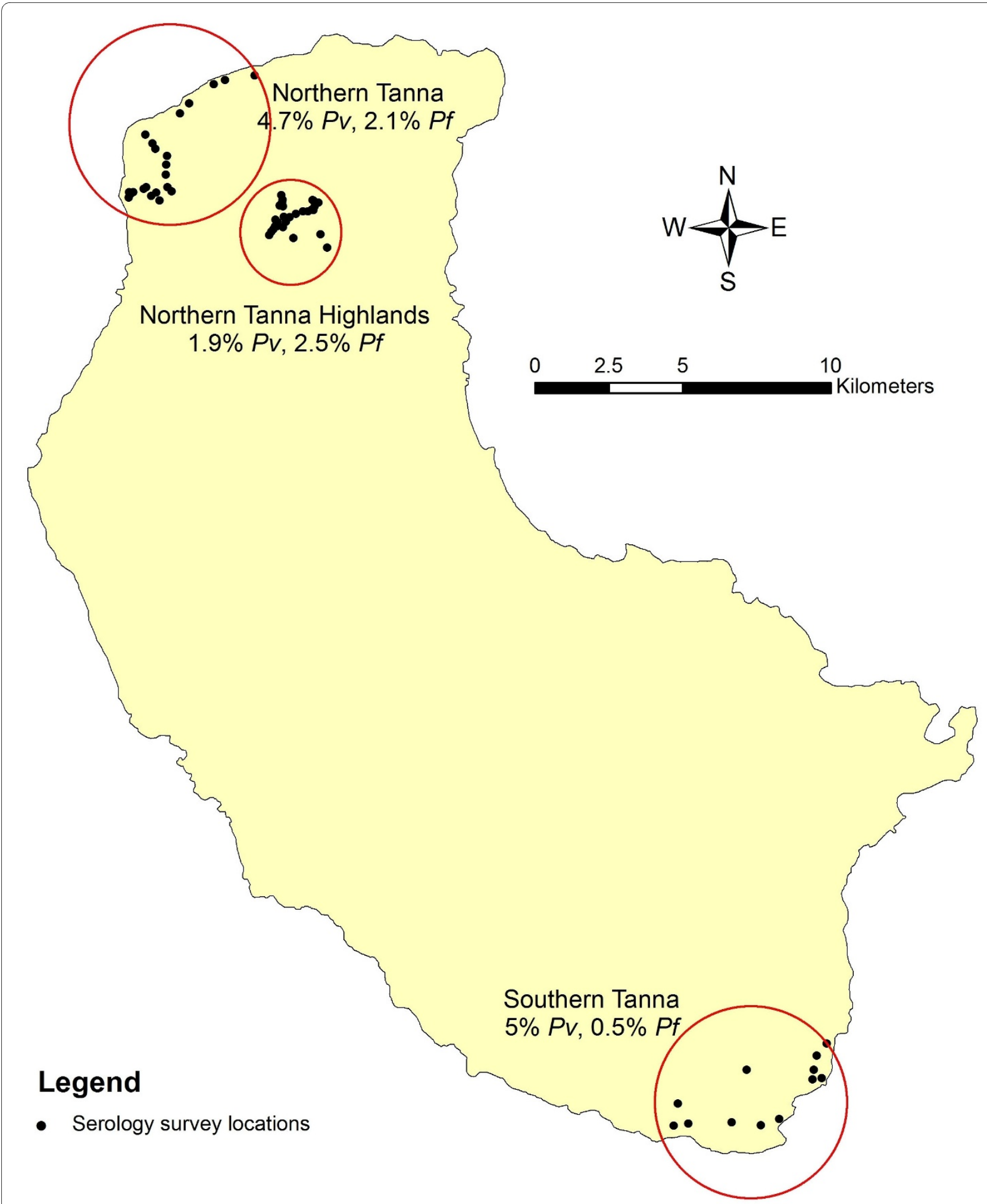

Figure 2 Summary of Plasmodium speciation PCR results obtained from Tanna in 2008. PCR results for children over 2 and under 15 are presented, adapted from data presented in Reid et al [22]. Each black spot represents the home settlement of at least one child involved in the survey. Children were sampled from a total of 44 villages across three settlements on Tanna. 


\section{Survey methodology}

The cross-sectional survey took place in May 2009. Participants were recruited to the study by the Ni-Vanuatu malaria control team. Village leaders were sensitized to the study by trained field workers and together provided information to community members at community meetings. Community members willing to participate were asked to complete a written Informed Consent Form by providing a signature or witnessed thumbprint. A central meeting point was chosen (nakamal, or a school or community centre) and families were invited from surrounding villages to be part of the survey. A cross-section of the population was sampled, with high levels of community participation. Demographic data were collected for each person, including age, sex and details on whether they owned a bed net, and had used a bed net the previous night. Participants were tested with a rapid diagnostic test (ICT COMBO cassette) if they presented with a fever and were treated accordingly if the test was positive. Finger prick blood samples were collected on labelled Whatman $3 \mathrm{~mm}$ filter paper, allowed to air dry and then packaged in sealable bags containing silica gel, before being transported for analysis in Brisbane, Australia.

\section{Assay of anti-malarial antibodies}

Antibodies were eluted from filter paper blood spots (FPBS) and assayed by ELISA as previously described [19]. Briefly, a $3.5 \mathrm{~mm}$ circle was cut from the spot and placed into $300 \mu \mathrm{l}$ of phosphate buffered saline with $0.5 \%$ tween 20 (PBS-T) and $0.05 \%$ sodium azide, approximately equivalent to a $1 / 200$ serum dilution. All samples were tested for the presence of human antibodies (IgG) against merozoite surface protein- $1_{19}\left(\right.$ MSP- $\left._{19}\right)$ and apical membrane antigen-1 (AMA-1) of P. falciparum (MSP- $1_{19^{-}}$ Wellcome strain, AMA-1-3D7) and P. vivax (MSP-1 $1_{19}$ Belem strain, AMA-1 (Sal-1). Samples were also tested on freeze thawed $P$. falciparum Schizont Extract (concentration of $1 \times 10^{8} / \mathrm{ml}$ ) which was coated onto plates at $1 / 500$. Briefly, each antigen was coated on high absorbance plates (Immunlon4) at $0.5 \mu \mathrm{g} / \mathrm{ml}$ and stored at $4^{\circ} \mathrm{C}$ overnight. The plate was washed three times in PBS-T and blocked for 3 hours with $1 \%(\mathrm{w} / \mathrm{v})$ milk powder. Following three more washes, samples were added in duplicate at a final dilution of $1 / 1000$. A pool of sera from a highly endemic area in Africa was titrated on each plate as a positive control. The following day, following five washes, anti-human IgG HRP (DAKO) was incubated in each plate for three hours. The plates were then developed using OPD (Sigmafast) for 20 minutes in the dark. Plates were read at $492 \mathrm{~nm}$ on a Molecular Devices Versa Max ELISA reader.

\section{Statistical methods}

The serology survey data was double entered into separate Microsoft Excel spreadsheets. The completed spreadsheets were converted to Microsoft Access databases which were compared using the FLG comparison script [25] which yielded $1.67 \%$ differences in entry that were corrected and checked again. The resulting double entered database was logically validated for value homogeneity and range checking, using scripts developed in SAS/STAT software (v1.93 for Windows). The cleaned database was then converted back to Microsoft Excel for analysis in STATA (v.10).

Samples where duplicate optical densities (ODs) had more than $20 \%$ variation between them were excluded from the analysis. Raw ODs were transformed into titres using the standard curve to normalize between plates and dichotomized into positive or negative using a mixture model, as described previously [19]. Similar models have been used for defining cut offs for the Mantoux test for tuberculosis where pre-exposure to environmental bacteria can cause non-specific reactions [26] and also in serological tests for measles and rubella $[27,28]$. Briefly, the data were fitted using maximum likelihood methods to establish seronegative and seropositive Gaussian distributions. For each antigen, the cut off was then set as the mean titre of the seronegative distribution plus three standard deviations [20].

A simple reversible catalytic model was fitted to the dichotomized data using maximum likelihood methods [18]. The model generates a seroconversion rate (SCR or $\lambda$ ) and a seroreversion rate $(\rho)$. For the purposes of this study the seroreversion rate was not fixed and allowed to vary as determined by the model. The model was used to generate age seroprevalence curves, from which a seroconversion rate (SCR) representing the force of infection for the community was calculated. If visual examination of SCR suggested it was not uniform over the whole population (i.e. there was an obvious step in the age seroprevalence data), models allowing for two forces of infection profile and profile likelihood plots were run to determine when the most likely time for change in transmission intensity occurred (or at what age the step in seroprevalence occurs) [20]. These resulted in a predicted time of change in transmission (and 95\% confidence intervals), which was subsequently incorporated into the catalytic model to generate estimates for previous and current SCR. Models allowing for two forces of infection were preferred if the fit compared to the single force model was significantly better by likelihood ratio (LR) test at a $\mathrm{p}$ $<0.05$.

The PfSE antigen is multi-antigenic and not necessarily specific for $P$. falciparum antigens as many of the same proteins are likely to be present in other species of Plasmodium and possibly in other infections. Therefore, the analysis primarily focuses on the results for the specific antigens analysed. Village of residence was mapped based on coordinates recorded the previous year [22]. Mean seroprevalence in children (to represent recent and local 
transmission) aged between 1 and 10 (inclusive) was plotted by village using ArcGIS (v.9.3.1). Logistic regression was used to compare differences in seroprevalence between settlements, whilst controlling for age group.

\section{Ethical approval}

This research was approved by the Vanuatu Ethics Committee, Ministry of Health, Vanuatu and by the University of Queensland.

\section{Results}

A total of 364, 515 and 370 people were recruited from North Tanna, the Northern Tanna Highlands and Southern Tanna respectively. Five hundred and seventeen people were recruited from Aneityum from two of the three villages. Fewer men were sampled than women (854/906) but this difference was not significant, and the ratio did not vary across clusters $\left(\mathrm{Chi}^{2} \mathrm{p}=0.87\right)$. Sampling often took place in schools, meaning that children of school age were more heavily sampled- the age distribution varied slightly depending on the cluster (Table 1). In total, 65\% of people sampled were under the age of 20 , a figure that is higher than the 55\% national distribution [29].

Due to current malaria control activities, ownership and reported use of bed nets was generally high, with usage close to $80 \%$ in Northern Tanna, Southern Tanna and Aneityum (Table 1). However in the Northern highlands in Tanna the reported usage of nets was lower, with only $38 \%(167 / 436)$ of survey respondents reporting to have used the nets the night before despite 98\% (503/512) reporting they owned nets (Table 1 ). This is likely to be a result of the perceived low malaria risk in this area[23].

\section{Antibody responses in the population}

Seroprevalence for each settlement, by age group, is summarized in Table 2 and Figure 3. Overall, seroprevalence increased with age and was higher to AMA-1 antigens than to MSP-1 19 antigens. Seroprevalence was highest to PfSE. There was no difference in seroprevalence between males and females for any of the antigens tested ( $p>0.1$ for all antigens). Overall, seropositivity in Tanna was $9.4 \%$ and $12.4 \%$ and $16.6 \%$ for P. falciparum MSP- $1_{19}$, AMA-1 and PfSE respectively and $12.6 \%$ and $15.0 \%$ for $P$. vivax MSP- $1_{19}$ and AMA- 1 respectively. In Aneityum, seropositivity was $6.6 \%, 5.8 \%$ and $11.8 \%$ for $P$. falciparum MSP$1_{19}$, AMA-1 and PfSE respectively and $6.2 \%$ and $10.1 \%$ for P. vivax $\mathrm{MSP}-1_{19}$ and AMA-1 respectively.

The seroprevalence data suggest that residents in Northern Tanna have experienced the highest exposure to P. falciparum malaria infection, with $22 \%$ (79/358) of individuals being seropositive to one or both of the specific P. falciparum antigens tested. Southern Tanna had the lowest specific P. falciparum seroprevalence (5\%, 20/ 369). P. vivax seroprevalence was also highest in Northern Tanna (30\%, 107/361), followed by Southern Tanna (25\%, 92/369), with the lowest seroprevalence in the Northern Tanna Highlands (11\%, 54/512).

Figure 3 demonstrates the increasing seroprevalence from PfMSP- $1_{19}$ to PfAMA with the highest seroprevalence to PfSE in each settlement. Interestingly, children

Table 1: Population demographics of survey participants (\%(n))

\begin{tabular}{|c|c|c|c|c|c|c|}
\hline & & $\begin{array}{c}\text { Northern } \\
\text { Tanna } \\
(n=364)\end{array}$ & $\begin{array}{c}\text { Northern Tanna } \\
\text { Highlands } \\
(\mathbf{n}=\mathbf{5 1 5})\end{array}$ & $\begin{array}{c}\text { Southern } \\
\text { Tanna } \\
(n=370)\end{array}$ & $\begin{array}{c}\text { Aneityum } \\
(n=517)\end{array}$ & $\begin{array}{c}\text { Total } \\
(n=1766)\end{array}$ \\
\hline & Male & $47.3(172)$ & $48.1(245)$ & $50.3(186)$ & $48.6(251)$ & $48.5(854)$ \\
\hline \multirow[t]{4}{*}{ Sex } & Female & $52.8(192)$ & $51.9(264)$ & 49.7 (184) & $51.5(266)$ & $51.5(906)$ \\
\hline & Not recorded & 0 & 6 & 0 & 0 & 6 \\
\hline & $0-5$ & $18.4(67)$ & 23.1 (119) & $14.1(52)$ & $17.4(90)$ & $18.6(328)$ \\
\hline & $5-20$ & $48.4(176)$ & $42.9(221)$ & 52.7 (195) & $42.4(219)$ & $45.9(811)$ \\
\hline \multirow[t]{3}{*}{ Age (Years) } & $20-80$ & $33.2(121)$ & $34.0(175)$ & $33.2(123)$ & $40.2(208)$ & $35.5(627)$ \\
\hline & Total & $100(364)$ & $100(515)$ & $100(370)$ & $100(517)$ & 100 (1766) \\
\hline & No & $21.8(79)$ & $61.7(269)$ & $11.1(41)$ & $10.1(52)$ & $26.1(441)$ \\
\hline \multirow{2}{*}{$\begin{array}{c}\text { Used a bed net the } \\
\text { night before the } \\
\text { survey }\end{array}$} & Yes & $78.2(284)$ & $38.3(167)$ & $88.9(329)$ & $89.9(465)$ & $73.8(1245)$ \\
\hline & Not recorded & 1 & 79 & 0 & 0 & 80 \\
\hline
\end{tabular}


Table 2: Seroprevalence to four malarial antigens by age group

\begin{tabular}{|c|c|c|c|c|c|c|c|c|c|c|c|c|c|c|c|c|}
\hline Location & $\begin{array}{c}\text { Age } \\
\text { group } \\
\text { (years) }\end{array}$ & $\begin{array}{l}\text { N Pf } \\
\text { MSP }\end{array}$ & $\begin{array}{l}\text { Pf MSP } \\
+ \text { ve }\end{array}$ & $\%$ Pf MSP +ve & $\begin{array}{l}\text { N Pf } \\
\text { AMA }\end{array}$ & $\begin{array}{l}\text { Pf AMA } \\
\text { +ve }\end{array}$ & $\begin{array}{c}\text { \% Pf AMA } \\
\text { +ve }\end{array}$ & N Pf SE & $\begin{array}{l}\text { Pf SE } \\
+ \text { ve }\end{array}$ & $\%$ PfSE +ve & $\begin{array}{l}\text { N Pv } \\
\text { MSP }\end{array}$ & 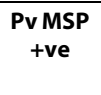 & $\%$ Pv MSP +ve & N Pv AMA & $\begin{array}{l}\text { Pv AMA } \\
\text { +ve }\end{array}$ & \% Pv AMA+ve \\
\hline & $0-5$ & 64 & 4 & $6.3(1.6-12.3)$ & 65 & 7 & $10.8(3.0-18.5)$ & 66 & 5 & $7.6(1.0-14.1)$ & 66 & 13 & $19.7(9.8-29.5)$ & 65 & 8 & $12.3(4.1-20.5)$ \\
\hline \multirow{4}{*}{$\begin{array}{l}\text { Northern } \\
\text { Tanna }\end{array}$} & $5-20$ & 176 & 15 & $8.5(4.4-12.7)$ & 174 & 29 & $16.7(11.1-22.3)$ & 176 & 25 & $14.2(9.0-19.4)$ & 172 & 27 & $15.7(10.2-21.2)$ & 175 & 20 & $11.4(6.7-16.2)$ \\
\hline & $20-80$ & 121 & 44 & $36.4(27.7-45.1)$ & 119 & 43 & $36.1(27.4-45.0)$ & 120 & 68 & $56.7(47.7-65.7)$ & 119 & 37 & $31.1(22.7-39.5)$ & 121 & 41 & $33.9(25.3-42.4)$ \\
\hline & Total & 361 & 63 & $17.5(13.5-21.4)$ & 358 & 79 & $22.1(17.8-26.4)$ & 362 & 98 & $27.1(22.5-31.7)$ & 357 & 77 & $21.6(17.3-25.9)$ & 361 & 69 & $19.1(15.0-23.2)$ \\
\hline & $0-5$ & 115 & 3 & $2.6(0-5.6)$ & 119 & 5 & $4.2(0.5-7.9)$ & 119 & 1 & $0.8(0.0-2.5)$ & 117 & 6 & $5.1(1.1-9.2)$ & 119 & 4 & $3.4(0.1-6.6)$ \\
\hline \multirow{4}{*}{$\begin{array}{l}\text { Northern } \\
\text { Tanna } \\
\text { Highlands }\end{array}$} & $5-20$ & 216 & 13 & $6.0(2.8-9.2)$ & 218 & 14 & $6.4(3.1-9.7)$ & 221 & 12 & $5.4(2.4-8.4)$ & 216 & 14 & $6.5(3.2-9.8)$ & 220 & 15 & $6.8(3.5-10.2)$ \\
\hline & $20-80$ & 173 & 21 & $11.6(7.2-17.1)$ & 174 & 35 & $20.1(14.1-26.1)$ & 176 & 66 & 37.9 (30.6-45.2) & 174 & 12 & $6.9(3.1-10.7)$ & 173 & 22 & $12.7(7.7-17.7)$ \\
\hline & Total & 504 & 37 & $7.3(5.1-9.6)$ & 511 & 54 & $10.6(7.9-13.2)$ & 514 & 79 & $15.4(12.2-18.5)$ & 507 & 32 & $6.3(4.2-8.4)$ & 512 & 41 & $8.0(5.6-10.4)$ \\
\hline & $0-5$ & 52 & 1 & $1.9(0-5.8)$ & 52 & 2 & $3.8(0-9.3)$ & 51 & 0 & 0.0 & 51 & 5 & $9.8(1.4-18.3)$ & 52 & 8 & $15.4(5.2-25.5)$ \\
\hline \multirow{4}{*}{$\begin{array}{c}\text { Southern } \\
\text { Tanna }\end{array}$} & $5-20$ & 195 & 3 & $1.5(0-3.3)$ & 188 & 7 & $3.6(1.0-6.2)$ & 192 & 3 & $1.6(0.0-3.3)$ & 195 & 22 & $11.3(6.8-15.8)$ & 194 & 31 & $16.0(10.8-21.2)$ \\
\hline & $20-80$ & 123 & 12 & $9.8(4.4-15.1)$ & 122 & 11 & $9.0(3.9-14.2)$ & 121 & 26 & $21.5(14.1-28.9)$ & 123 & 19 & $15.5(9.0-21.9)$ & 122 & 37 & $30.3(22.1-38.6)$ \\
\hline & Total & 370 & 16 & $4.3(2.2-6.4)$ & 349 & 20 & $5.4(3.1-7.7)$ & 364 & 29 & $8.0(5.2-10.8)$ & 369 & 46 & $12.5(9.1-15.9)$ & 368 & 76 & $20.7(16.5-24.8)$ \\
\hline & $0-5$ & 90 & 1 & $1.1(0-3.3)$ & 90 & 4 & $4.4(0.1-8.8)$ & 90 & 4 & $4.4(0.1-8.7)$ & 90 & 3 & $3.3(0-7.1)$ & 88 & 3 & $3.4(0-7.3)$ \\
\hline \multirow[t]{3}{*}{ Aneityum } & $5-20$ & 218 & 9 & $4.1(1.5-6.8)$ & 218 & 10 & $4.6(1.8-7.4)$ & 219 & 5 & $2.3(0.3-4.3)$ & 219 & 9 & $4.1(1.5-6.8)$ & 210 & 12 & $5.7(2.6-8.9)$ \\
\hline & $20-80$ & 208 & 24 & 11.5 (7.2-15.9) & 208 & 16 & $7.7(4.0-11.1)$ & 208 & 52 & $25(19.1-30.9)$ & 208 & 20 & $9.6(5.6-13.7)$ & 207 & 36 & $17.4(12.2-22.6)$ \\
\hline & Total & 516 & 34 & $6.6(4.4-8.7)$ & 516 & 30 & $5.8(3.8-7.8)$ & 517 & 61 & $11.8(9.0-14.6)$ & 517 & 32 & $6.2(4.1-8.3)$ & 505 & 51 & $10.1(7.5-12.7)$ \\
\hline
\end{tabular}

Number of positive individuals and seroprevalence ( $95 \%$ Cls) to PfMSP- $1_{19}$, PfAMA, PfSE, PvMSP- ${ }_{19}$ and PvAMA for three age groups in three settlements on Tanna and two settlements combined on Aneityum 


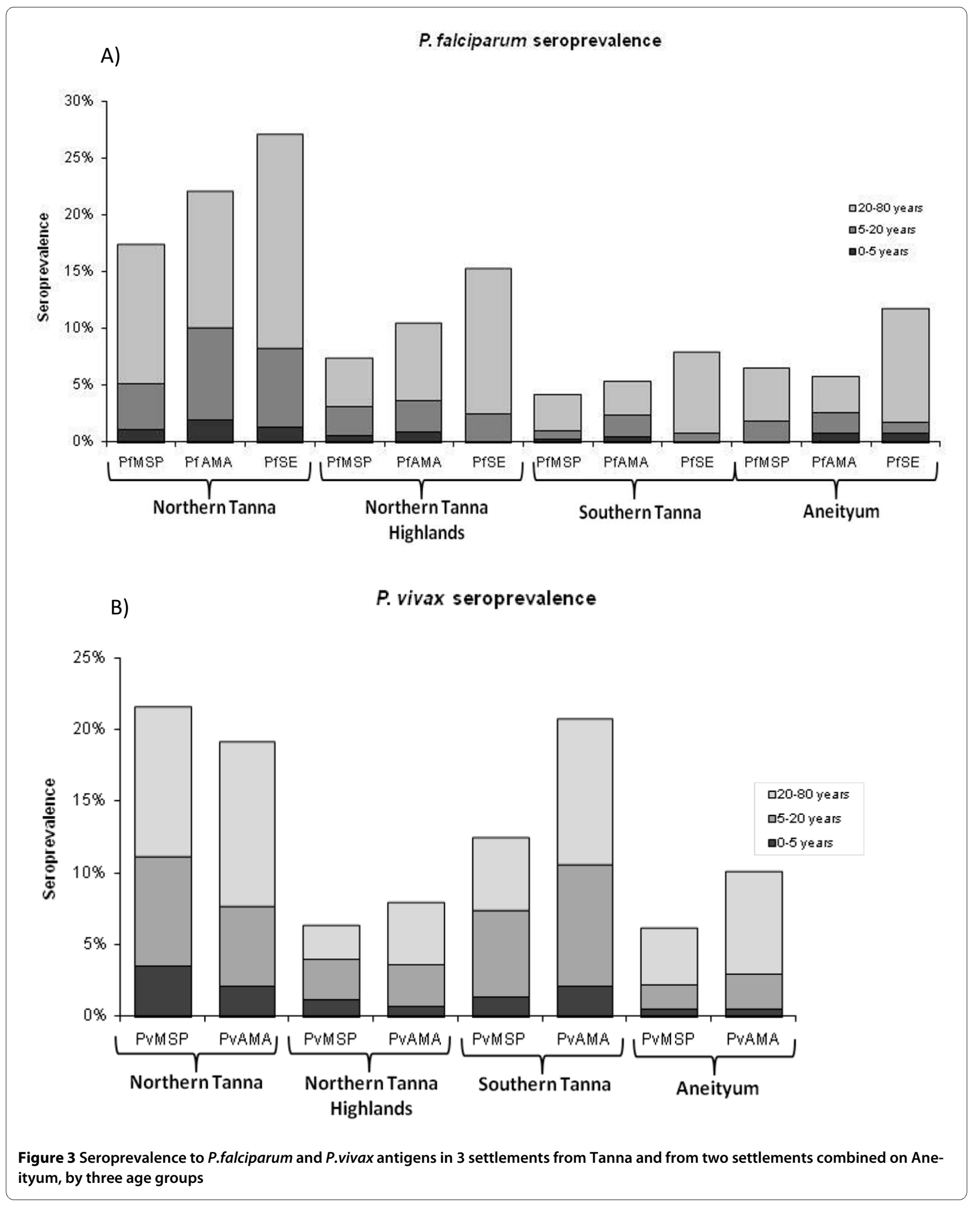

under five consistently had lower seroprevalence to PfSE than to the specific antigens. This contrasts with adults who had the highest seroprevalence to PfSE in all settlements.
Seroprevalence to specific antigens in children between the ages of 1 and 10 years (inclusive) by village is shown in Figure 4. It suggests that exposure is not uniform across the settlements with focal areas becoming evident in 
North Tanna and Southern Tanna. Unfortunately numbers were too small to allow for micro-spatial analysis.

\section{Changes in transmission intensity over time}

Changes in transmission intensity were identified most readily for PfSE, with weak evidence in responses to the more specific PfMSP- $1_{19}$ seroprevalence (Figure 5). No clear peaks were identified for PfAMA or for the two $P$. vivax antigens. For PfMSP- $1_{19}$, the PLPs did not show strong evidence for a change in transmission, as demonstrated by the log likelihoods all being above the $95 \%$ confidence intervals (Figure 5) however, the MSP- $1_{19}$ PLPs generally peaked at the same point as the PfSE plots. The maximum $\log$ likelihoods indicate that a reduction in $P$. falciparum transmission intensity occurred approximately 30 years ago in Northern Tanna (PfSE PLP: 33 years (95\% CI: 27-50) and the North Tanna Highlands (PfSE PLP: 28 years (22-32 years)), 23 years ago in Aneityum (PfSE PLP: 23 years (21-32 years)) and approximately 18 years ago in Southern Tanna (PfSE PLP: 18 years (9-28 years)). The resulting SCRs suggest a 3, 9 and 12 fold decrease in transmission for North Tanna, North Tanna Highlands and Southern Tanna respectively (Figure 6). Transmission on Aneityum appears to have decreased over seven-fold. Although the decrease in transmission is not reflected in the age seroprevalence data for the specific antigens, the SCRs are very low for all settlements.
Peaks were less clear in PLP for seropositivity to $P$. vivax antigens, suggesting that $P$. vivax transmission may not have undergone a distinct reduction. A PvSE antigen was not available at the time of processing; it is possible that evidence for a change in transmission would have been detected with this antigen, as was seen with the $P$. falciparum equivalent.

On Aneityum, it appeared that changes in age seroprevalence were more readily detected in population PfSE antibodies. The PfSE log likelihood indicated a change occurred approximately 23 years ago (18-38 years); the PfMSP- $1_{19}$ PLP indicated a change occurred between 25 and 31 years ago, although this was not significant (Figure 7). The PvAMA seroprevalence curve for Aneityum (Figure 7) showed some evidence for a change in force of infection, although this was not reflected in the fitted model.

\section{Evidence for elimination?}

The current SCRs for all antigens for both species were very low in Aneityum. Only the PfSE seroprevalence curve showed evidence of a clear change-point (Figure 6). However, in areas where malaria is thought to be eliminated, it is pertinent to investigate individual positive responses. Seroprevalence to specific malarial antigens in Aneityum was 10\% (54/516) and 13\% (65/505) for P. falciparum and $P$. vivax specific antigens respectively. Seropositive status was mainly restricted to older individuals. However, six children under 7 were seropositive for one

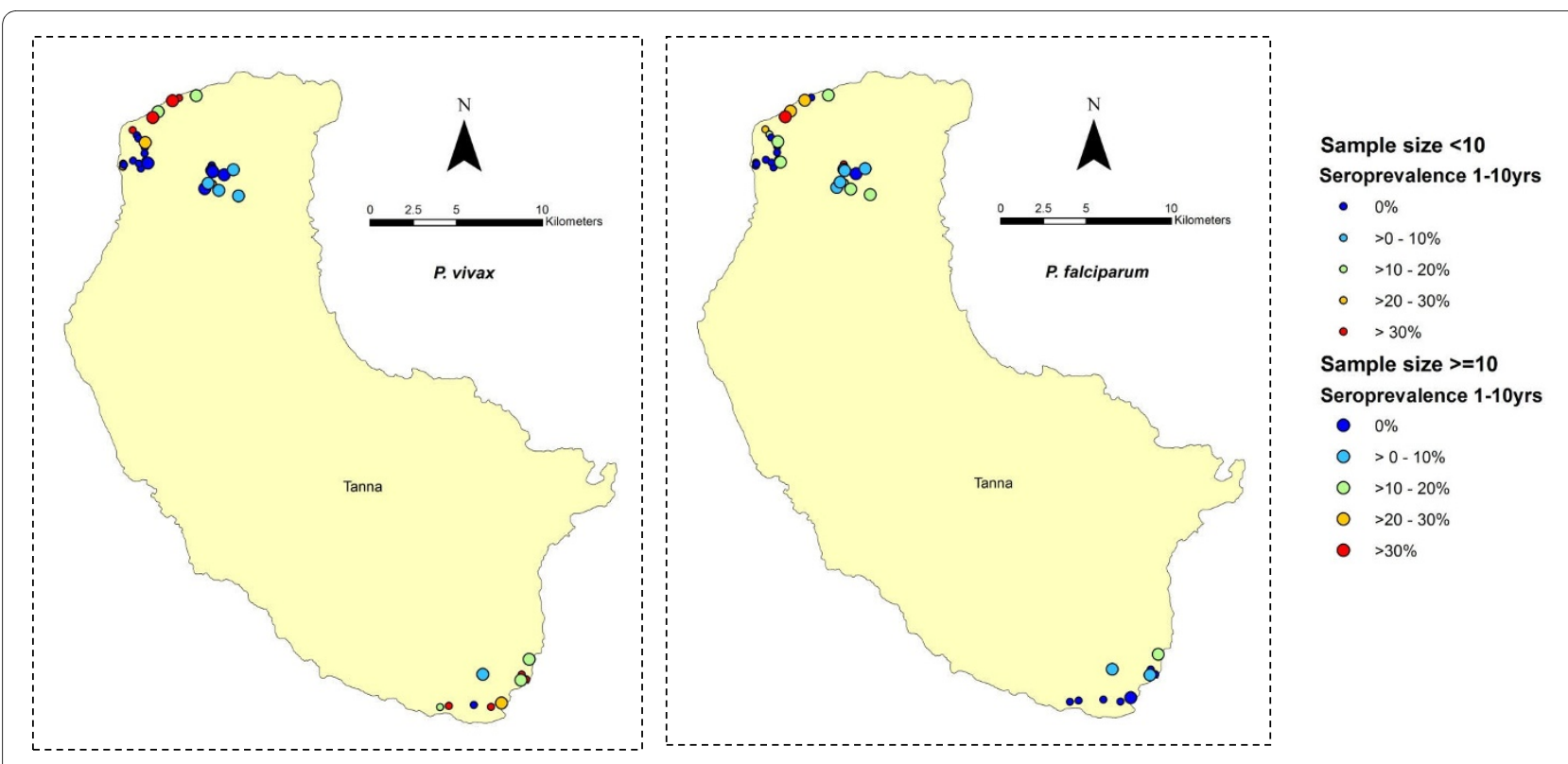

Figure 4 The distribution of seroprevalence in children aged 1-10 (inclusive) in settlements on Tanna. Seroprevalence to specific $P$. falciparum antigens (MSP-1 19 and/or AMA-1) in children aged 1-10 (inclusive) is plotted in figure $4 \mathrm{a}$ and the equivalent for P.vivax in Figure $4 \mathrm{~b}$. Young children were chosen to represent local transmission as they are less likely to have travelled to areas of higher transmission. In some cases, under 10 children were sampled from a single village- these are represented by smaller circular symbols, whilst the larger symbols represent villages where more than ten, and up to 48 children were sampled. 
a)

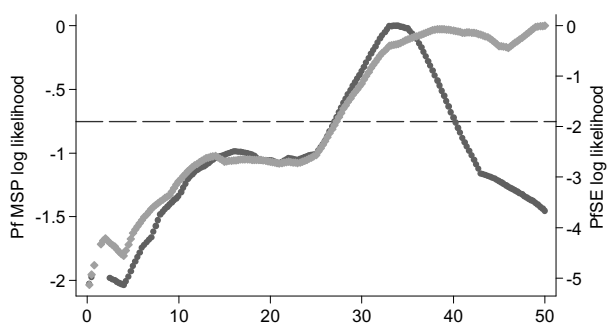

b)

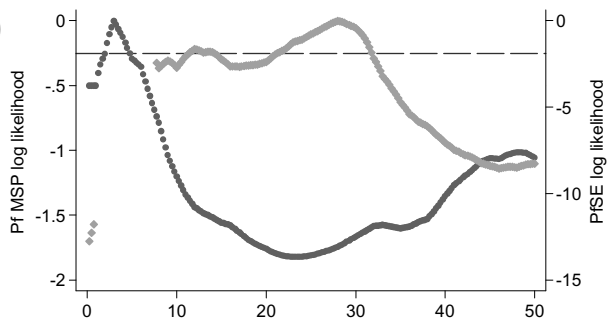

c)

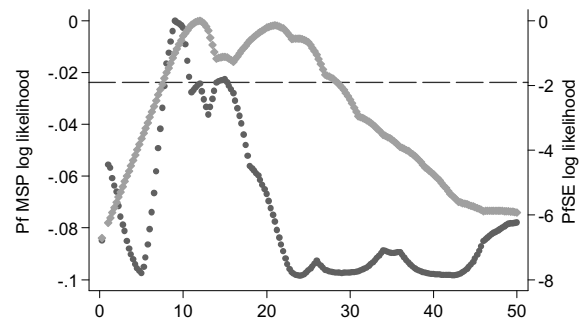

d)

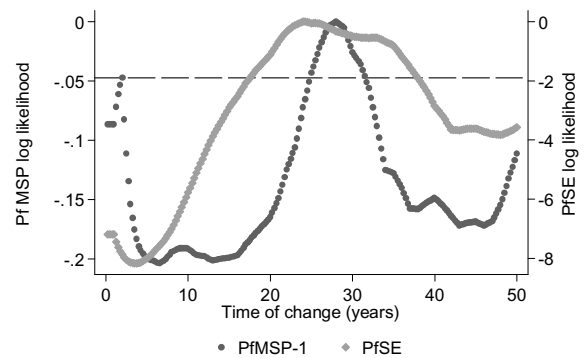

Figure 5 Profile likelihood plots (PLPs) for PfMSP-1 ${ }_{19}$ and PfSE for a) Northern Tanna b) Northern Tanna Highlands c) Southern Tanna and d) Aneityum. Profile likelihood plots (PLPS) show the log likelihood of a catalytic conversion model allowing for a change in transmission occurring at iterative years. The maximum log likelihood is the time point at which a change in transmission is most likely to have occurred. Plots for PfAMA and the two $P$. vivax antigens showed no discernible peaks. The plots for Northern Tanna and Aneityum suggest a change in transmission occurred approximately 30 years ago, whilst the PLP for Southern Tanna suggests a more recent change between 10 and 20 years ago.

or both $P$. falciparum antigens and five children under 7 were seropositive to one or both $P$. vivax antigens. Two of the children positive for $P$. falciparum antigens were reported to be one years old and antibodies may have been of maternal origin. Two children, aged 2 and aged 6, were positive to all antigens tested for both species.

The Northern settlement of Port Patrick on Aneityum had a higher proportion of seropositive individuals. Twenty-nine percent (20/70) of the individuals tested from Port Patrick were seropositive to $P$. falciparum specific antigens and $25 \%(17 / 68)$ were seropositive to $P$. vivax antigens, compared to $8 \%(34 / 446)$ and $11 \%(48 /$ $437)$ in Anel. When controlled for age group, people in Port Patrick were 5 times more likely to be $P$. falciparum seropositive and twice as likely to be $P$. vivax seropositive. Thirty-three percent (4/12) of the under $7 \mathrm{~s}$ (born since the 2002 epidemic) in Port Patrick were positive for $P$. falciparum antigens compared to $2 \%(2 / 91)$ of under $7 \mathrm{~s}$ in 


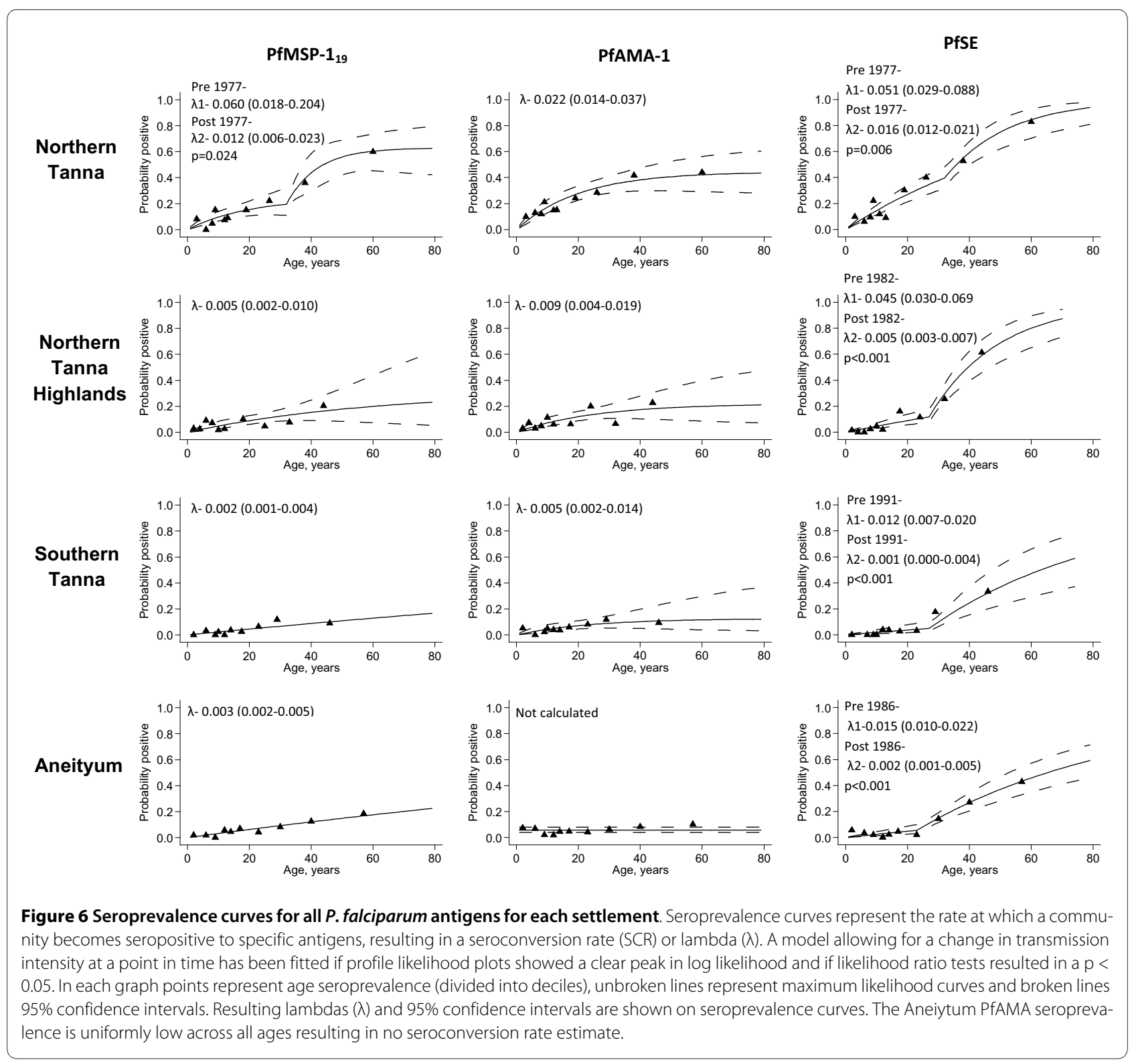

Anel ( $\mathrm{p}<0.001)$, while 20\% (2/9) were positive for $P$. vivax antigens compared to 3\% (3/90) in Anel ( $<<0.001)$. There was no evidence of clinical infection on the island during the survey.

\section{Discussion}

The Vanuatu government aims to eliminate malaria from Tafea province (which includes Tanna \& Aneityum) in the next six years. It is thought that malaria has already been eliminated from Aneityum [3] and that transmission is confined to specific locations across Tanna. In this study, the applicability of using serological methods to provide current estimates of transmission intensity was assessed and the utility of serology to provide evidence for the cessation of transmission in Aneityum was also investigated.
Seroepidemiological studies are especially useful in low transmission settings where the sensitivity of parasite prevalence surveys is limited by the scarcity of parasite positive individuals. This study was undertaken in the dry season, and as such only three RDT positives $(0.2 \%$ of the population surveyed) were found in the population. In the previous wet season, surveys using PCR methods in the same areas on Tanna found 1\% prevalence of P. falciparum and $2.2 \%$ prevalence of $P$. vivax infection in children between 2-11 years old [22]. Both parasite-based prevalence measures, which offer a snapshot of malaria prevalence and are influenced by temporal changes, are, not surprisingly, significantly lower than the antibody prevalence data (which offers a period prevalence measure) for Tanna in this study $(24 \%, 299 / 1242$ P. falciparum 


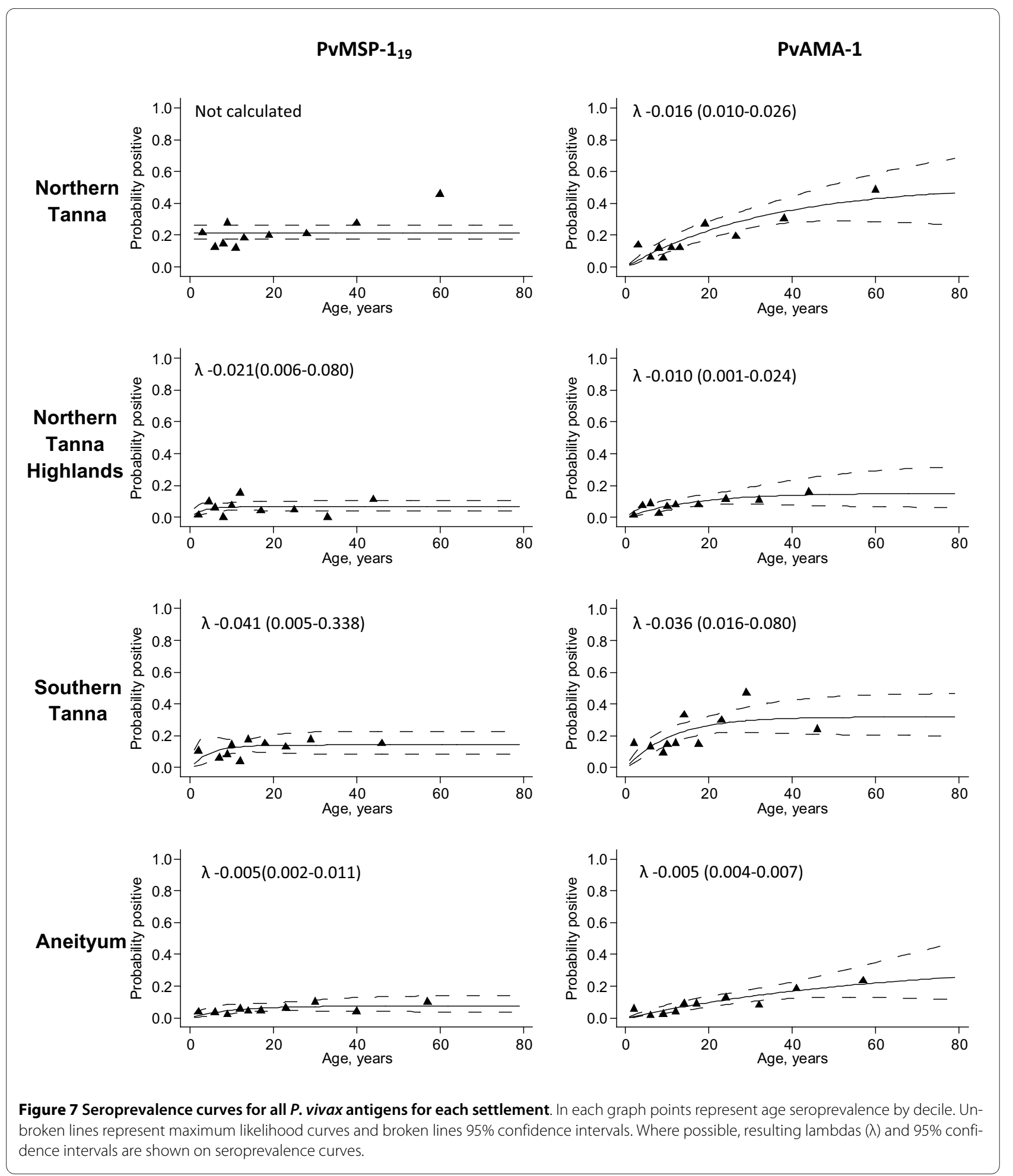

and 20\%, 253/1242 P. vivax). This extra sensitivity allows for a more detailed examination of malaria transmission.

Modelling changes in transmission intensity can give an insight into the success of interventions in a particular area. Perhaps surprisingly, changes in transmission were most readily detected using PfSE. Schizont extract con- tains multiple proteins some of which will be antigenic and similar between parasite species, it is, therefore, not surprising that PfSE has the highest overall seroprevalence. However, whilst adults demonstrate high seroprevalence to PfSE, seroprevalence in children is lower than for the more specific antigens, MSP- $1_{19}$ and AMA-1. It is 
not clear why this should be; it may be the result of lower exposure in children resulting in lower levels of antibodies to the antigens in PfSE. This has been demonstrated in Kenya, where individuals were more likely to have antibodies to specific antigens rather than PfSE [30]. An alternative explanation is that the relative concentration of the specific antigens is lower in PfSE- this needs further investigation.

Statistical analysis of the seroprevalence profiles suggests that a significant drop in transmission occurred approximately 30 years ago. These seroprevalence curves are consistent with those described by Kaneko et al (submitted) using a P. falciparum lysate. However, analysis of country-wide case data suggests that a drop in malaria cases occurred in 1992 (17 years before this study), which was attributed to ITN distribution across the islands [24]. There are two (not mutually exclusive) potential explanations for this. Firstly, the drop in seroprevalence may indicate that the IRS campaign in place in the early 1980s successfully reduced exposure. At the time, this campaign was considered unsuccessful due to little reduction exhibited in national parasite rates. However, it is possible that it was effective in certain areas such as Northern Tanna and on Aneityum. An alternative explanation is that the reduction in seroprevalence is related to the later malaria control programme that was implemented nationwide in 1988 and in Aneityum in 1991, and which resulted in the elimination of malaria from Aneityum [3]. The observation that the step in serological data occurs approximately 10 years before this point in this scenario is possibly due to differential rates of loss of antibodies with age. Younger children who had low antibody levels when the 1988 interventions were implemented, may have lost these antibodies more rapidly than older individuals with a more established antibody response $[31,32]$. Clearly more information on the rates of antibody loss is required to allow a full interpretation of these data, and further work is ongoing.

The observation that immune responses to the specific antigens do not reflect the changes in transmission is perplexing. Transmission is known to be very low across Tanna and it is possible that in these conditions models for assessing for an increase in seropositivity with age may be inappropriate. Several of the age stratified curves demonstrate little to no increase in seroprevalence with age. In areas where transmission has been consistently low for many years a loss in seropositivity in older individuals may result in uniform seropositivity across the population; masking any change-point in transmission. This is clearly not the case for the multi-antigenic PfSEone might expect an increased chance that antibodies to this conglomeration of antigens have longer half lives, although this was not observed in a longitudinal study in Thailand [33]. In a situation where age seroprevalence reveals little information, it may be more pertinent to investigate antibody titres and to focus on children born since interventions have been in place. This work is ongoing.

It is important to understand the dynamics in the transmission of different species in areas where more than one species of Plasmodium are transmitted. Serological measures have allowed us to successfully distinguish between areas where $P$. falciparum and $P$. vivax malaria are transmitted. Current SCRs are low for both species but consistently higher for $P$. vivax in all areas. Seropositivity to $P$. vivax antigens was much higher in younger individuals, suggesting either that transmission of this species has been higher than P. falciparum transmission over the past 10 years, or that preferential infection of younger age groups, as has been reported previously [34], results in a different age seroprevalence curve than is found with $P$. falciparum seroprevalence. Additionally, morbidity data from previous studies in Vanuatu [35] and Papua New Guinea [36] show that clinical immunity to $P$. vivax builds up faster than for $P$. falciparum which could explain the seemingly high seroprevalence in younger children. The high proportion of $P$. vivax seropositive individuals in younger age groups in Northern Tanna made the data difficult to fit the conventional seroconversion model. A similar age seroprevalence profile would be expected in areas where there has been an epidemic as the result of a defined period of exposure in all ages of the population. However, the effect of possible presence of hypnozoites in $P$. vivax infections has not previously been studied and we do not know how infections that are the result of hypnozoites (i.e. relapses) influence serological profiles. Previous data has shown that $P$. falciparum is more successfully reduced by interventions than $P$. vivax, due to the limited effects interventions have on relapses, and due to differences in biting behaviour by mosquitoes infected with each species [37]. The profile likelihood plots do not indicate a clear change in transmission intensity based on seroprevalence of $P$. vivax antibodies. The drop in $P$. vivax transmission may not have been as pronounced or sudden as the $P$. falciparum reduction, in which case, a step in the seroprevalence curves would not be evident. $P$. vivax prevalence tends to be higher in areas of low transmission intensity [38], and an increase in $P$. vivax cases has been reported in areas where $P$. falciparum transmission appears to be on the decline $[39,40]$. However, on Aneityum clear evidence of a change in transmission for $P$. vivax was seen in seroprevalence data by Kaneko et al (submitted); this data also indicated a change in transmission had occurred in the early 1980s. This study used a lysed P.vivax preparation as antigen which, as for the $P$. falciparum equivalent, may be more likely to detect changes in a polymorphic antibody response. 
The $P$. vivax epidemic in 2002 may also have had an adverse effect on the seroprevalence curves to $P$. vivax antigens in Aneityum. There have been no reported cases since 2002, and whilst seropositivity on this island is mainly restricted to adults, low seropositivity in children under 7 suggests that there may still be residual transmission in the area. However, importantly, four of the 11 children seropositive to any antigen (falciparum or vivax) were classified as aged one. Birthdays and ages are not generally recorded in Aneityum, and it is possible these children were younger than one and, therefore, the antibodies detected could represent residual maternal antibodies. Alternatively, children may have been exposed to malaria whilst away from the island. Limited information was collected on travel history during the survey so this possible explanation cannot be confirmed. Monitoring of cases on Aneityum is very thorough, with everyone returning to the island tested for infection via thick and thin slide films. However, slide reading has limited sensitivity, and it is possible that some people may have arrived carrying infection. PCR methods detect parasites more sensitively and it may be pertinent to check for subpatent infections amongst the inhabitants of Aneityum. Imported malaria can remain a problem as long as Anopheline mosquitoes are present on the island. This highlights the importance of continued monitoring and surveillance on islands where elimination has been achieved [41], and the recording of accurate travel history information.

An alternative explanation for the observed seropositivity in young children is the presence of antibodies cross-reacting with antigens from other infectious agents [42-44]. Detecting antibodies to specific recombinant antigens using ELISA, rather than the less specific immunofluoresence antibody test (IFAT) minimizes this danger. However, a few studies have suggested the crossreactive potential of PfAMA with Toxoplasma antigens $[45,46]$, although this infection is reportedly not prevalent in Vanuatu.

\section{Conclusion}

Malaria transmission is notably heterogeneous, and this will become more pronounced as transmission decreases. For malaria elimination, areas where residual transmission is occurring need to be identified in order to target interventions to communities where they are most needed. In Vanuatu, interventions have been very successful at reducing MTI over the past 20 years. However, areas of higher transmission were identified on Tanna during the 2008 survey [22]. Serological analysis and monitoring of these areas can offer further insight into the dynamics of malaria transmission. The additional sensitivity afforded by the longevity of antibody responses also allows an examination of the success of control measures also in confirming the cessation of transmission. Serological measures are robust and high throughput and importantly, remain sensitive in very low transmission settings.

\section{Competing interests}

The authors declare that they have no competing interests.

\section{Authors' contributions}

$J C$ carried out the survey, the serological experiments, and the serological analysis and wrote the first draft of the manuscript. HR developed the study protocol, led the field survey and produced the maps for the serological analysis with support from AC. Jl participated in field operations, data and laboratory specimen collection and conducted serological assays. MK supported field team operations, coordinated laboratory procedures and serological testing. GT conceived the study, participated in its design and supervised field coordination. JM, AV, AC and CD conceived the study, participated in its design and implementation and supervised fieldwork, laboratory investigations, data analysis and manuscript development. All authors read and approved the final manuscript.

\section{Acknowledgements}

We are extremely grateful for the expertise of the Ni-Vanuatu team who helped to undertake this survey and would like to thank all the participants involved, without whom the work would not have been possible. We would also like to thank Gerry Kelly and his team for mapping the villages and informing inhabitants of the impending study. We thank Teun Bousema for comments and guidance with the writing of the manuscript, Eleanor Riley and Patrick Corran for interpretation of the data analysis and Akira Kaneko for sharing his expertise from many years of working in Vanuatu. We would additionally like to thank Tony Holder, Bart Faber and Robin Anders for the kind donation of antigens, or their constructs.

\section{Author Details}

'London School of Hygiene \& Tropical Medicine, Keppel Street, London WC1E 7HT, UK, 2 School of Population Health, University of Queensland, Herston, Qld 4006, Australia, ${ }^{3}$ Ministry of Health, Port Vila, Vanuatu, ${ }^{4}$ Queensland Institute of Medical Research, Herston, Qld 4006, Australia and 5 School of Medicine, University of Queensland, Herston, Qld 4006, Australia

Received: 23 February 2010 Accepted: 16 June 2010 Published: 16 June 2010

\section{References}

1. Feachem RGA, Phillips AA, Targett GA: Shrinking the Malaria Map: A prospectus on Malaria Elimination The Global Health Group; 2009.

2. Bousema T, Drakeley C, Gesase S, Hashim R, Magesa S, Mosha F, Otieno S, Carneiro I, Cox J, Msuya E, Kleinschmidt I, Maxwell C, Greenwood B, Riley E, Sauerwein R, Chandramohan D, Gosling R: Identification of hot spots of malaria transmission for targeted malaria control. J Infect Dis 2010, 201:1764-1774.

3. Kaneko A, Taleo G, Kalkoa M, Yamar S, Kobayakawa T, Bjorkman A: Malaria eradication on islands. Lancet 2000, 356:1560-1564.

4. Teklehaimanot HD, Teklehaimanot A, Kiszewski A, Rampao HS, Sachs JD: Malaria in Sao Tome and principe: on the brink of elimination after three years of effective antimalarial measures. Am J Trop Med Hyg 2009, 80:133-140

5. Bruce-Chwatt LJ, Draper CC, Konfortion P: Seroepidemiological evidence of eradication of malaria from Mauritius. Lancet 1973, 2:547-551.

6. Smith DL, Hay SI: Endemicity response timelines for Plasmodium falciparum elimination. Malar J 2009, 8:87

7. Kelly-Hope LA, McKenzie FE: The multiplicity of malaria transmission: a review of entomological inoculation rate measurements and methods across sub-Saharan Africa. Malar J 2009, 8:19.

8. Robert V, Le Goff G, Andrianaivolambo L, Randimby FM, Domarle O, Randrianarivelojosia M, Raharimanga V, Raveloson A, Ravaonjanahary C, Ariey F: Moderate transmission but high prevalence of malaria in Madagascar. Int J Parasitol 2006, 36:1273-1281.

9. Elissa N, Migot-Nabias F, Luty A, Renaut A, Toure F, Vaillant M, Lawoko M, Yangari P, Mayombo J, Lekoulou F, Tshipamba P, Moukagni R, Millet P, 
Deloron P: Relationship between entomological inoculation rate, Plasmodium falciparum prevalence rate, and incidence of malaria attack in rural Gabon. Acta Trop 2003, 85:355-361.

10. Corran P, Coleman P, Riley E, Drakeley C: Serology: a robust indicator of malaria transmission intensity? Trends Parasitol 2007, 23:575-582.

11. Voller A, Bruce-Chwatt $\mathrm{L}$ : Serological malaria surveys in Nigeria. Bull World Health Organ 1968, 39:883-897.

12. Cornille-Brogger R, Mathews HM, Storey J, Ashkar TS, Brogger S, Molineaux L: Changing patterns in the humoral immune response to malaria before, during, and after the application of control measures: a longitudinal study in the West African savanna. Bull World Health Organ 1978, 56:579-600.

13. Voller A, Cornille-Brogger R, Storey J, Molineaux L: A longitudinal study of Plasmodium falciparum malaria in the West African savannah using the ELISA technique. Bull World Health Organ 1980, 58:429-438.

14. Draper CC, Lelijveld JL, Matola YG, White GB: Malaria in the Pare area of Tanzania. IV. Malaria in the human population 11 years after the suspension of residual insecticide spraying, with special reference to the serological findings. Trans R Soc Trop Med Hyg 1972, 66:905-912.

15. Ambroise-Thomas P, Wernsdorfer WH, Grab B, Cullen J, Bertagna P: Etude sero-epidemiologique longitudinale sur la paludisme en Tunisie. Bull Organ Mond Sante 1976, 54:355-367.

16. Bruce-Chwatt LI, Draper CC, Avramidis D, Kazandzoglou O: Seroepidemiological surveillance of disappearing malaria in Greece. J Trop Med Hyg 1975, 78:194-200.

17. Drakeley C, Cook J: Chapter 5. Potential contribution of seroepidemiological analysis for monitoring malaria control and elimination: historical and current perspectives. Adv Parasitol 2009, 69:299-352.

18. Drakeley C, Corran P, Coleman PG, Tongren JE, McDonald S, Carneiro I, Malima R, Lusingu JPA, Manjurano A, Nkya WM, Lemnge MM, Cox J, Reyburn $\mathrm{H}$, Riley EM: Estimating medium and long term trends in malaria transmission using serological markers of malaria exposure. Proc Natl Acad Sci USA 2005, 102:5108-5113.

19. Corran PH, Cook J, Lynch C, Leendertse H, Manjurano A, Griffin J, Cox J, Abeku T, Bousema T, Ghani AC, Drakeley C, Riley EM: Dried blood spots as a source of anti-malarial antibodies for epidemiological studies. Malar J 2008, 7:195.

20. Stewart L, Gosling R, Griffin J, Gesase S, Campo J, Hashim R, Masika P, Mosha J, Bousema T, Shekalaghe S, Cook J, Corran P, Ghani A, Riley EM, Drakeley C: Rapid assessment of malaria transmission using agespecific sero-conversion rates. PLoS One 2009, 4:e6083.

21. Williams GS, Mweya C, Stewart L, Mtove G, Reyburn H, Cook J, Corran PH, Riley EM, Drakeley CJ: Immunophoretic rapid diagnostic tests as a source of immunoglobulins for estimating malaria sero-prevalence and transmission intensity. Malar $J$ 2009, 8:168.

22. Reid H, Vallely A, Taleo G, Tatem AJ, Kelly G, Riley I, Harris I, lata H, Yama S, Clements $\mathrm{A}$ : Baseline spatial distribution of malaria prior to an elimination program in Vanuatu. Malar $J 2010$ in press.

23. Atkinson JA, Fitzgerald L, Toaliu H, Taleo G, Tynan A, Whittaker M, Riley I, Vallely A: Community participation for malaria elimination in Tafea Province, Vanuatu: Part I. Maintaining motivation for prevention practices in the context of disappearing disease. Malar J 2010, 9:93

24. Chaves LF, Kaneko A, Taleo G, Pascual M, Wilson ML: Malaria transmission pattern resilience to climatic variability is mediated by insecticidetreated nets. Malar J 2008, 7:100.

25. Gray DJ, Forsyth SJ, Li RS, MCManus DP, Li Y, Chen H, Zheng F, Williams GM: An innovative database for epidemiological field studies of neglected tropical diseases. PLoS Negl Trop Dis 2009, 3:e413.

26. Nagelkerke NJ, Borgdorff MW, Kim SJ: Logistic discrimination of mixtures of M. tuberculosis and non-specific tuberculin reactions. Stat Med 2001, 20:1113-1124.

27. Hardelid P, Williams D, Dezateux C, Tookey PA, Peckham CS, Cubitt WD, Cortina-Borja M: Analysis of rubella antibody distribution from newborn dried blood spots using finite mixture models. Epidemiol Infect 2008:1-9.

28. Rota MC, Massari M, Gabutti G, Guido M, De Donno A, Atti ML: Measles serological survey in the Italian population: Interpretation of results using mixture model. Vaccine 2008, 26:4403-4409.

29. SPC: Secretariat of the Pacific Community: Oceania Population Data Sheet. 2000.
30. Osier FH, Fegan G, Polley SD, Murungi L, Verra F, Tetteh KK, Lowe B, Mwangi T, Bull PC, Thomas AW, Cavanagh DR, McBride JS, Lanar DE, Mackinnon MJ, Conway DJ, Marsh K: Breadth and magnitude of antibody responses to multiple Plasmodium falciparum merozoite antigens are associated with protection from clinical malaria. Infect Immun 2008, 76:2240-2248.

31. Akpogheneta OJ, Duah NO, Tetteh KK, Dunyo S, Lanar DE, Pinder M, Conway DJ: Duration of naturally acquired antibody responses to blood-stage Plasmodium falciparum is age dependent and antigen specific. Infect Immun 2008, 76:1748-1755.

32. Kinyanjui SM, Conway DJ, Lanar DE, Marsh K: IgG antibody responses to Plasmodium falciparum merozoite antigens in Kenyan children have a short half-life. Malar J 2007, 6:82.

33. Wipasa J, Suphavilai C, Okell LC, Cook J, Corran PH, Thaikla K, Liewsaree W, Riley EM, Hafalla JC: Long-lived antibody and B cell memory responses to the human malaria parasites, Plasmodium falciparum and Plasmodium vivax. PLoS Pathog 2010, 6:e1000770.

34. Price RN, Tjitra E, Guerra CA, Yeung S, White NJ, Anstey NM: Vivax malaria: neglected and not benign. Am J Trop Med Hyg 2007, 77:79-87.

35. Maitland K, Williams TN, Bennett S, Newbold Cl, Peto TE, Viji J, Timothy R, Clegg JB, Weatherall DJ, Bowden DK: The interaction between Plasmodium falciparum and $P$. vivax in children on Espiritu Santo island, Vanuatu. Trans R Soc Trop Med Hyg 1996, 90:614-620.

36. Lin E, Kiniboro B, Gray L, Dobbie S, Robinson L, Laumaea A, Schopflin S, Stanisic D, Betuela I, Blood-Zikursh M, Siba P, Felger I, Schofield L, Zimmerman $P$, Mueller I: Differential patterns of infection and disease with P. falciparum and P. vivax in Young Papua New Guinean children. PLoS One 2010, 5:e9047.

37. Bockarie MJ, Dagoro H: Are insecticide-treated bednets more protective against Plasmodium falciparum than Plasmodium vivax-infected mosquitoes? Malar J 2006, 5:15

38. Mendis K, Sina BJ, Marchesini P, Carter R: The neglected burden of Plasmodium vivax malaria. Am J Trop Med Hyg 2001, 64:97-106.

39. Childs DZ, Cattadori IM, Suwonkerd W, Prajakwong S, Boots M: Spatiotemporal patterns of malaria incidence in northern Thailand. Trans R Soc Trop Med Hyg 2006, 100:623-631.

40. da Silva-Nunes M, Codeco CT, Malafronte RS, da Silva NS, Juncansen C, Muniz PT, Ferreira MU: Malaria on the Amazonian frontier: transmission dynamics, risk factors, spatial distribution, and prospects for control. Am J Trop Med Hyg 2008, 79:624-635.

41. D'Ortenzio E, Sissoko D, Dehecq JS, Renault P, Filleul L: Malaria imported into Reunion Island: is there a risk of re-emergence of the disease? Trans R Soc Trop Med Hyg 2009.

42. Naus CW, Jones FM, Satti MZ, Joseph S, Riley EM, Kimani G, Mwatha JK, Kariuki CH, Ouma JH, Kabatereine NB, Vennervald BJ, Dunne DW: Serological responses among individuals in areas where both schistosomiasis and malaria are endemic: cross-reactivity between Schistosoma mansoni and Plasmodium falciparum. J Infect Dis 2003, 187:1272-1282

43. Mutapi F, Roussilhon C, Mduluza T, Druilhe P: Anti-malaria humoral responses in children exposed to Plasmodium falciparum and Schistosoma haematobium. Mem Inst Oswaldo Cruz 2007, 102:405-409.

44. Abramo C, Fontes CJ, Krettli AU: Cross-reactivity between antibodies in the sera of individuals with leishmaniasis, toxoplasmosis, and Chagas' disease and antigens of the blood-stage forms of Plasmodium falciparum determined by indirect immunofluorescence. Am J Trop Med Hyg 1995, 53:202-205.

45. Donahue CG, Carruthers VB, Gilk SD, Ward GE: The Toxoplasma homolog of Plasmodium apical membrane antigen-1 (AMA-1) is a microneme protein secreted in response to elevated intracellular calcium levels. Mol Biochem Parasitol 2000, 111:15-30.

46. Gaffar FR, Yatsuda AP, Franssen FF, de Vries E: Erythrocyte invasion by Babesia bovis merozoites is inhibited by polyclonal antisera directed against peptides derived from a homologue of Plasmodium falciparum apical membrane antigen 1. Infect Immun 2004, 72:2947-2955.

doi: 10.1186/1475-2875-9-169

Cite this article as: Cook et al., Using serological measures to monitor changes in malaria transmission in Vanuatu Malaria Journal 2010, 9:169 